\title{
A Nonlinear TSNN Based Model of a Lead Acid Battery
}

\author{
El Mehdi Laadissi, Anas El Filali, Malika Zazi
}

Laboratory LM2PI, ENSET, Mohamed V University, Rabat, Morocco

\begin{tabular}{l} 
Article Info \\
\hline Article history: \\
Received Aug 31, 2017 \\
Revised Apr 30, 2018 \\
Accepted May 14, 2018 \\
\hline Keywords: \\
ANN \\
Hammerstein-wiener \\
Lead acid battery \\
NARX \\
TSNN \\
\hline
\end{tabular}

\begin{abstract}
The paper studies a nonlinear model based on time series neural network system (TSNN) to improve the highly nonlinear dynamic model of an automotive lead acid cell battery. Artificial neural network (ANN) take into consideration the dynamic behavior of both input-output variables of the battery charge-discharge processes. The ANN works as a benchmark, its inputs include delays and charging/discharging current values. To train our neural network, we performed a pulse discharge on a lead acid battery to collect experimental data. Results are presented and compared with a nonlinear Hammerstein-Wiener model. The ANN and nonlinear autoregressive exogenous model (NARX) models achieved satisfying results.
\end{abstract}

Copyright (c) 2018 Institute of Advanced Engineering and Science. All rights reserved.

Corresponding Author:

El Mehdi Laadissi,

Laboratory Robotics and Control of Linear and Non-Linear Systems,

Mohamed V University,

Avenue des Nations Unies, Rabat 10000, Morocco.

Email: elmehdi.laadissi@um5s.net.ma

\section{INTRODUCTION}

A battery in its simplest form is a storage container of electricity for future use, an ideal battery would be able to deliver an infinite amount of current for an infinite amount of time and would work in any environment, but in reality, batteries are not ideal, they can't supply infinite power. The role of a lead acid battery in automotive applications for example is to start the engine by supplying power to the starter and ignition system plus to supply extra power when the vehicle's electrical load exceeds the supply from the alternator, and finally to act as a voltage stabilizer and dampening device in the electrical system. A 12-volt car battery consists of a plastic case with six cells connected in series, each producing two volts, each cell is made up of negative (sponge lead plates) and positive (lead oxide plates). The electrolyte is made up of a mix of thirty-five percent sulfuric acid and sixty-five percent water, the electrochemical reaction between the lead plates and the electrolyte produce DC electricity. During the discharge phase of the battery a by-product of the electrochemical reaction is the production of lead sulfate which coat the plates.

Recently, considerable attention has been paid to batteries. Modelling a battery is a very effective method for analyzing and simulating storage systems without the risk of aging them (renewable energy storage). The lead acid battery has a technology that is well mastered and almost entirely recyclable. It has the non-negligible advantage of presenting the lowest cost price among all known battery types. It plays, given its antiquity and its wide diffusion the role of benchmark to evaluate the characteristics of other batteries.

The literature on battery modelling shows a variety of approaches, several models have been developed. Until now, there is not a perfect model, because they do not take into consideration all parameters such as temperature and age of the battery, as well as some physical and chemical phenomena that occur inside the accumulator while the charging-discharging phases. In addition, some reduced models have been developed according to specific applications and in some cases for one type of battery. For example, a 
Chemical model is a complex model that considers the typically electrochemical phenomena involved such as diffusion, polarization and mass transfer within the electrochemical couple [1]-[3]. The chemical model consists of mathematical models represented by partially differential equations that are difficult to solve because they require initial and boundary conditions. In addition, these complex models require several chemical parameters which are difficult to determine. Another model is the empirical model, which is a classical method based on experimental tests. The performance of the battery is recorded and tabulated. This type of model does not represent a generic model for all the accumulators because it does not take into account all the internal parameters, it is necessary to make tests for each type of accumulator.

The simplest and most common model, illustrated in Figure 1, consists of an ideal voltage source $\mathrm{V}_{0}$ (no-load voltage) in series with an internal resistance. $\mathrm{V}_{\mathrm{t}}(\mathrm{t})$ is the terminal voltage across the battery. In this simple model $R_{i}$ and $V_{0}$ are considered constant. This model does not consider either the variation of the internal resistance of the accumulator as a function of the state of charge or of the temperature. This model can be applied if the dependence of the load state and temperature parameters can be overlooked [4]. However, researchers have arrived at the conclusion that the most accurate battery model is the "Nonlinear dynamic model". It is a variant of the Thevenin model that considers the non-linearity of the parameters. In this model, shown in Figure 2 below, the process of charging and discharging is separated. In addition, all parameters are dependent on the state of charge of the battery.

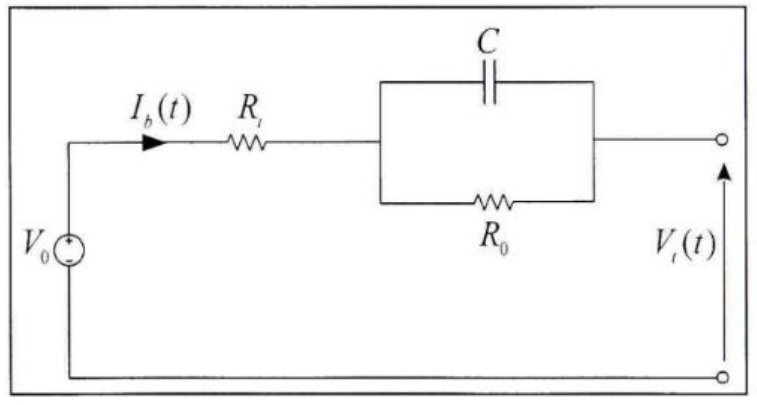

Figure 1. Equivalent circuit of Thevenin model

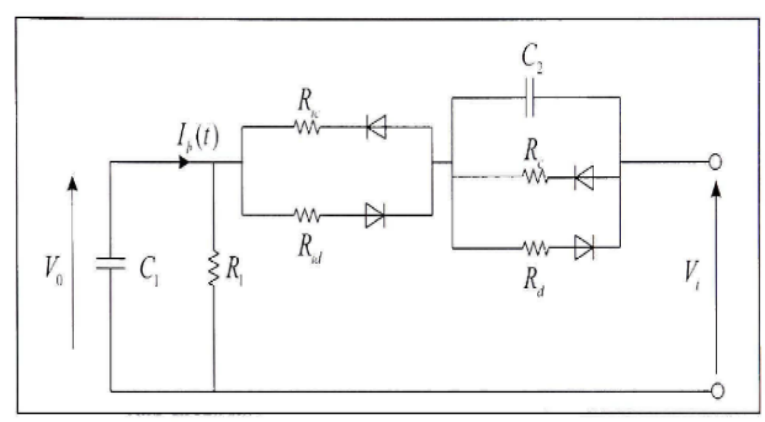

Figure 2. Nonlinear model of the battery

The parameters of the model are defined as follows:

$\mathrm{C}_{1}$ : Capacity of the accumulator.

$\mathrm{R}_{1}$ : Self discharge resistance.

$\mathrm{R}_{\mathrm{ic}}$ and $\mathrm{R}_{\mathrm{id}}$ : Represent the internal resistance due to the electrolyte and the electrodes respectively during charging and discharging.

The two $R C$ circuits $\left(R_{C} C_{2}\right.$ and $\left.R_{d} C_{2}\right)$ respectively represent the overvoltage at the end of the load and the sudden drop of the voltage at the end of the discharge. Since all the parameters of this model are variable (depending on the state of charge or the unloaded voltage), their identification is difficult.

As we have seen, there are different models in the literature [5][6], each of these models has its own characteristics. The model chosen depends on the application, if we want more precision then we choose a more detailed model taking into account all the parameters that can affect the performance of the lead acid battery. The nonlinear dynamic model is very interesting because it has a charge and discharge circuit and all parameters depend on the state of charge. The Cauer and Foster model [7]-[9] is used much more to represent the phenomenon of charge transfer and that of diffusion (chemical phenomena).

Extensive comparisons and evaluations with some selected models, generally recognized as reliable have shown that further efforts are needed to make refinements on the single battery cell model [1]-[12]. The paper proposes an improved approach based on the time series neural network, in order to obtain an improved discharge battery modelling using the neuro-procedure processing of the experimental voltage data using constant current discharge regime. A time series neural network (TSNN) can be usefully exploited to improve the battery modeling especially at low rate discharge regime. In some operating regimes (Fast charging/Deep discharging) underestimated and overestimated output values, depending on the simulated models, lead to failures in cell output voltage prediction. The TSNN model provides more accurate voltage values compared to the simple mathematical battery cell model, which makes it possible to obtain an improved low-rate voltage modeling. The main simulation results reported in the paper are compared with

BEEI, Vol. 7, No. 2, June 2018 : $169-175$ 
the experimental and a nonlinear Hammerstein-Wiener based model. The battery modeling was made based on time series neural network system to simulate it nonlinear behavior.

\section{EXPERIMENTAL SETUP}

Data collecting is considered as the first and essential part in identification terminology. In order to compare the presented methods, some battery data were gathered from a commercial automotive $62 \mathrm{Ah}, 12 \mathrm{~V}$, 540A (TUDOR TB620) lead acid battery. As input, a pulse-discharge test was performed. In this test, the battery was fully charged using constant current/constant voltage profile, then it was discharged using a $-4 \mathrm{~A}$ pulse current. A “ADS1299EEG-FE Data Acquisition" was used as data acquisition system (DAQ). The collected DATA are shown in Figure 3.
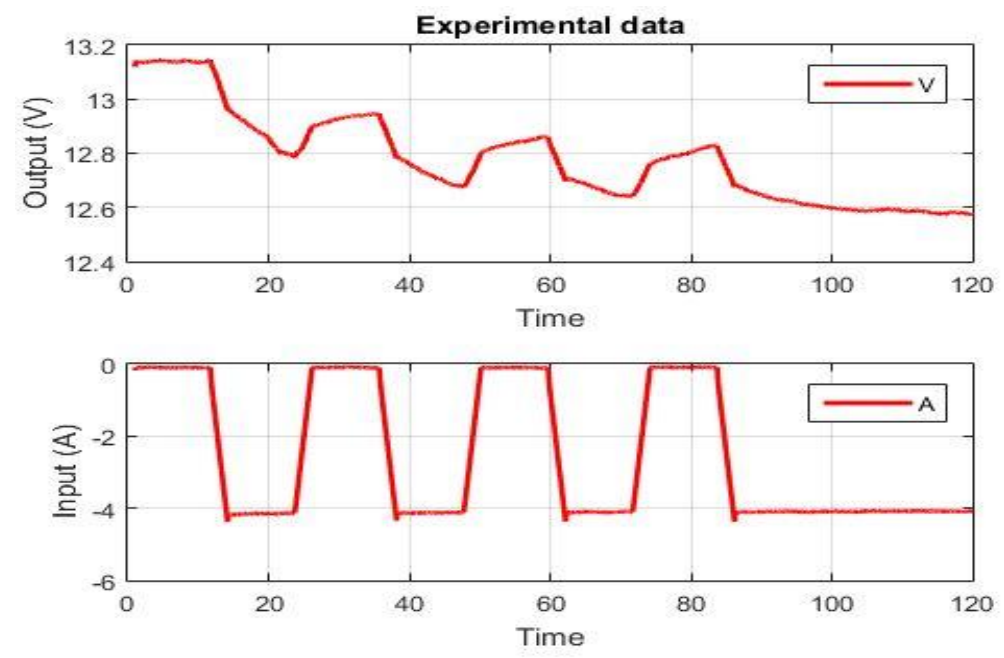

Figure 3. Experimental data

\section{CURRENT MODELS}

The current neural net models for lead acid battery output prediction makes use of the feed forward multi layered perceptron or back propagation algorithms to train the network [10]. Thus, this model cannot adequately account for the perturbation of the voltage output and thus provides a prediction with a lower accuracy. With such a configuration, the training phase of the network is faster, however the results sometimes show a greater deviation from the target.

\section{PROPOSED NARX NEURAL NETWORK MODEL ARCHITECTURE}

Nonlinear autoregressive exogenous model (NARX) neural network is a variant of Recurrent Network [11], [12] that has been successfully utilized in time series prediction problems. The major difference between recurrent neural network (RNN) and TSNN is that RNN allows a weighted feedback connection between layers of neurons and thereby making it suitable for time series analysis by allowing lagged values of variables to be considered in model. Although throughout the literature many time series methods such Autoregressive (AR), Moving Average (MA), Autoregressive Moving Average (ARMA), Autoregressive Integrated Moving Average (ARIMA), etc. have been applied in various econometrics problems, these techniques cannot cope with nonlinear problems. NARX on the contrary can efficiently be used for modelling non-stationary and nonlinear time series. Mathematically input output representation of nonlinear discrete time series in NARX network is governed by the following Equation 1. 


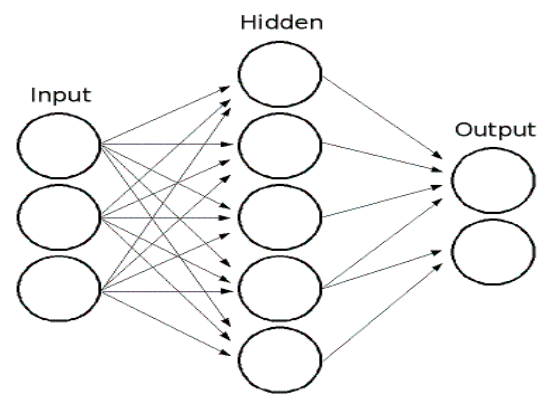

Figure 4. Current neural network model architecture

$$
(t)=[u(t-D u), \ldots, u(t-1), u(t), y(t-D y), . ., y(t-1)]
$$

where $u(t)$ and $g(t)$ represent input and output of the network at time t. Du and Dy, are the input and output order, and the function $\mathrm{f}$ is a nonlinear function. The function is approximated by TSNN. It is also possible to have NARX networks with zero input order and a one-dimensional output. i.e., having feedback from output only. In such cases operation of NARX network is governed by Equation 2.

$$
(t)=\Psi[u(t), y(t-1), \ldots(t-D)]
$$

where $\Psi$ is the mapping function, approximated by standard TSNN.

\subsection{Time Series Modelling}

The objective of time series forecasting is to predict future values of a time series, $\mathrm{Xn}+\mathrm{m}$ based on the observed data to present, $X=\{X n, X n-1, \ldots, X 1\}$. A lot of the time series forecasting model assume Xt to be stationary.

We have performed time series modeling of a lead acid battery using a NARX model. The used network is dynamic in nature as it has feedback loops (outputs of a neuron fed back to some previous neuron) and taps (delay lines that feed the network with past values of inputs). The use of a NARX network helps to increase the accuracy of prediction as well as can be used for real time prediction of state of charge (SOC) and state of health (SOF).

The model proposed here has one set of exogenous inputs which are the lead acid battery Current. The target series comprises of the voltage value of the lead acid battery. The output of the neural network is predicting the battery output current value over the entire target series along with the next value in the series. This is a curve-fitting problem which is implemented using MATLAB version 2015a. A parallel NARX network is created using "narxnet" command which helps in increasing the accuracy of the network. Also, here we have plotted the time series model of a black-box nonlinear model based on Hammerstein-Wiener of the same lead acid battery for comparison. For the network training phase, we used the "LevenbergMarquardt" algorithm which makes it possible to obtain a numerical solution to the problem of minimizing a function $\mathrm{F}(\mathrm{x})$ Equation (3), often nonlinear and dependent on several variables. The algorithm interpolates the Gauss-Newton algorithm and the gradient algorithm. More stable than that of Gauss-Newton, it finds a solution even if it is started very far from a minimum. However, for some very regular functions, it can converge slightly less quickly. The algorithm was developed by Kenneth Levenberg, then published by Donald Marquardt.

$$
F(x)=\frac{1}{2} \sum_{i=1}^{m}[f i(x)]^{2}
$$

\subsection{Results of TSNN Modelling}

The following Figure 5 present a sample NARX structure [13]-[15] which will be used in this paper. 


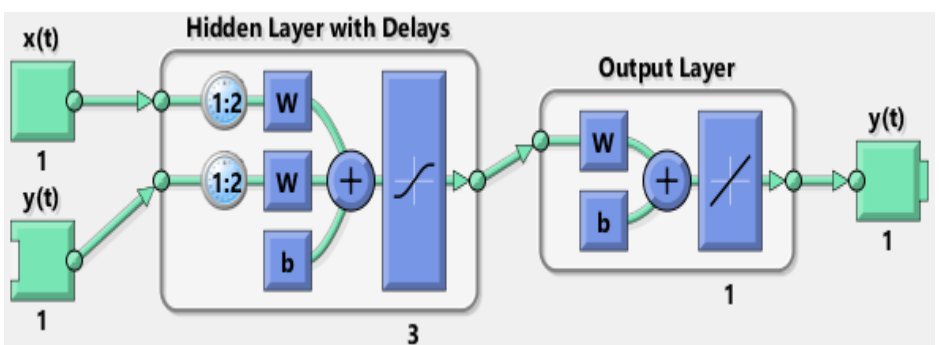

Figure 5. Simple NARX structure

Figure 6 graphically represents the association of the actual and predicted values for all training, test and validation samples. Magnitude of error expressed as difference between actual and predicted values is also shown in the same Figure. Although visual representation strongly suggests goodness of fit of NARX network in predicting output voltage, to quantitatively justify the claim, Mean-squared error (MSE) and regression $(\mathrm{R})$ values are computed for training and test dataset.

Statistics of MSE and R of predictive modelling performance of NARX network on training and test dataset for all experimental trials are summarized in following Table 1 and Table 2.

Table 1. Performance on Training Dataset

\begin{tabular}{cc}
\hline MSE & $\mathrm{R}$ \\
\hline $7.91567 \mathrm{e}-7$ & $9.99985 \mathrm{e}-1$ \\
\hline
\end{tabular}

Table 2. Performance on Test Dataset

MSE R

$7.91643 \mathrm{e}-7$

$9.99845 \mathrm{e}-1$

It is evident from negligible MSE and high $\mathrm{R}$ values that the presented NARX network with 2 delays units has predicted battery output voltage as a nonlinear function quite effectively. Error histogram with 20 bins is displayed in Figure 7.

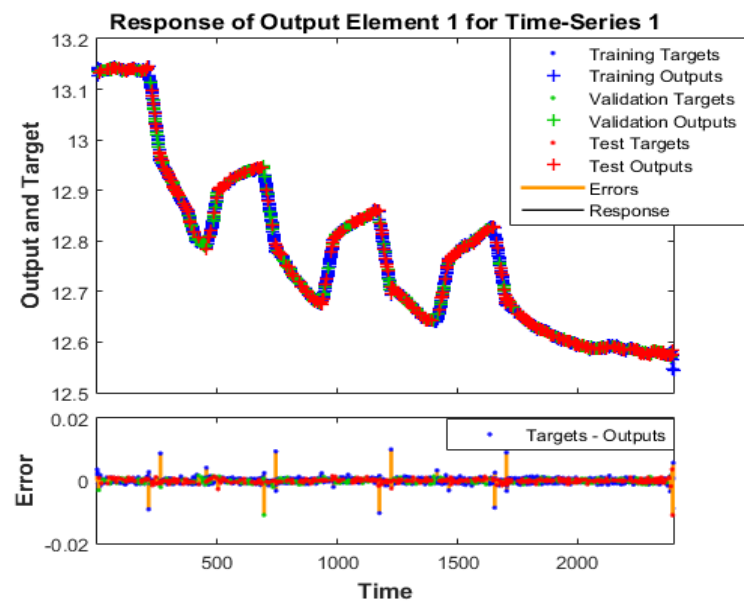

Figure 6. Visualization of NARX performance

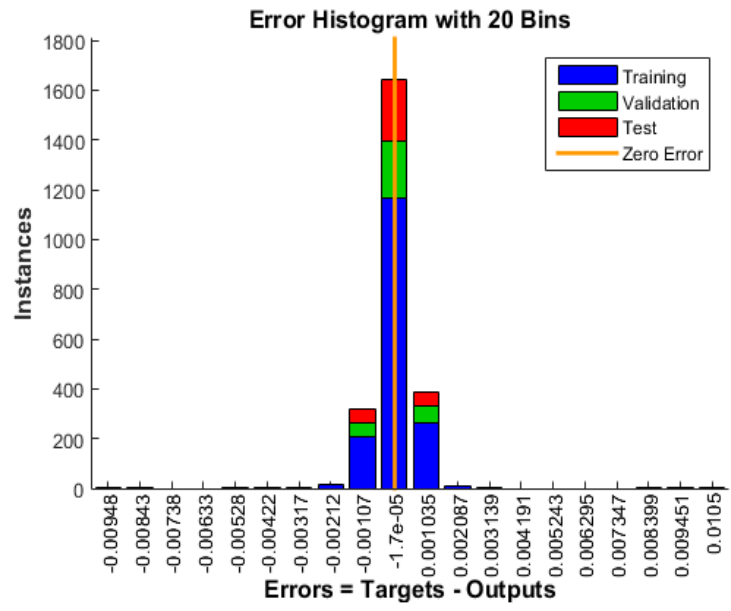

Figure 7. Error histogram of NARX modelling

\section{NONLINEAR HAMMERSTEIN-WIENER BASED MODEL}

When the output of a system depends nonlinearly on its inputs, sometimes it is possible to decompose the input-output relationship into two or more interconnected elements. In this case, we can represent the dynamics by a linear transfer function and capture the nonlinearities using nonlinear functions of inputs and outputs of the linear system. The Hammerstein-Wiener model achieves this configuration as a series connection of static nonlinear blocks with a dynamic linear block [16].

The number of units in the wavelet network and the sigmoid network was set to 20 . The value of the delay parameter and the number of units in the networks were purposefully varied in order to find the best configuration for our nonlinear model. 
The results in Figure 8 are obtained by processing experimental data obtained from the battery. "NL Model1" is the Hammerstein-Wiener model based on wavelet network as output nonlinearity. "NL Model2" is the Hammerstein-Wiener model based on sigmoid network as output nonlinearity.

Obviously in Figure 8 and Figure 9, "NL Model1" with a fit of 92.05\% outperformed "NL Model2" with a fit of $82.89 \%$ due to their difference in terms of output nonlinearity network structure. However, although the calculated weight functions for both the wavelet and sigmoid networks are optimal in the sense that they gave the best possible performance. These models must be able to adapt to changes in the ambient conditions or battery parameters.

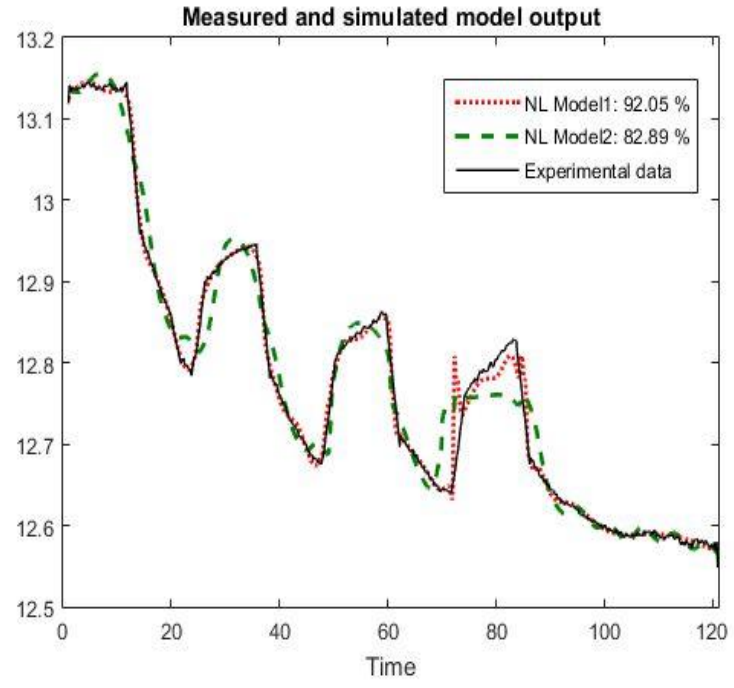

Figure 8. Measured and simulated model battery output

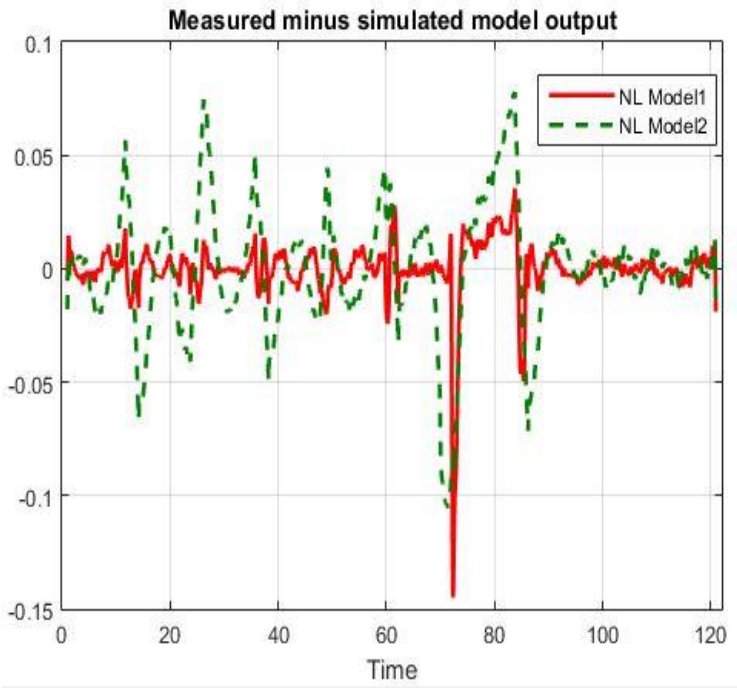

Figure 9. Measured minus simulated models output

\section{CONCLUSION \& FUTURE SCOPES}

The paper present both TSNN based model and Hammerstein-Wiener models in a multivariate framework to predict the output voltage of a lead acid battery. The study is based on real collected data. During the process of generating results, it was observed that both sets of techniques generated useful and efficient predictions of the voltage value. The application of both Hammerstein-Wiener and NARX including the use of various backpropagation algorithms is quite unique and the non-linear relationship between the input battery current and the output voltage have been effectively captured.

From the technique point of view, it is observed that the predictive performance of HammersteinWiener model does not depend on the number of neurons in the hidden layer (nonlinear output). For the NARX model, neither the number of neurons, nor the training algorithms, significantly affect the performance.

The proposed model can be incorporated into complex "Narxnet" models which can be used to predict the output voltage over a range of time periods or can be used to predict the state of health as well as the state of charge with high precision. Also, the accuracy of the model can be further increased by allowing the model to analyze and comprehend the trend of the exogenous input. Also, we can incorporate a fuzzy system through. The continuation of this work will be the implantation on an FPGA card to build a robust Battery management system "BMS".

\section{REFERENCES}

[1] Gu W, B Wang, C Y, Li, S M, Geng M \& Liaw B. Modeling discharge and charge characteristics of nickel-metal hydride batteries. Eletrochimica Acta. Volume 44, Numéro 25, 1999; 4525-4541.

[2] Osvaldo B, Francesco V \& Luigi G. State of charge Kalman filter estimator for automotive batteries. Control Engineering Practice, Volume 14, Numéro 3. 2006; 267-275.

[3] Fan D. \& White RE. A mathematical model of a sealed nickelcadmium battery. Journal of Electrochemistry Societ. 1991; Volumel38. Numéro 1. 
[4] Durr M. Cruden S. \& McDonald J.R. Dynamic model of a lead acid battery for use in a domestic fuel cell System. Journal of Power Sources, Volume 161, Numéro 2. 2006:1400-1411.

[5] Laadissi El, El Filali A \& Zazi M. Nonlinear Black Box Modeling of a Lead Acid Battery Using HammersteinWiener MODEL. Journal of Theoretical and Applied Information Technology. 2016; 89(2): 476.

[6] M Nandana Jyothi, V Dinakar, N S S Ravi Teja, K Nanda Kishore. NARX Based Short Term Wind Power Forecasting Model. TELKOMNIKA Indonesian Journal of Electrical Engineering. 2013; Vol 15 No 1.

[7] Kuhn E, Forgez C, Lagonotte P \& Friedrich G. Modelling Ni-Mh battery using Cauer and Foster structures. Journal of Power Sources. 2006; Volume 158. 2: 1490-1497.

[8] Jensimiriam E, Seenichamy P, \& Ambalavanan S. Prediction of lead-acid battery performance parameter: a neural network approach. Bulletin of Electrical Engineering and Informatics, 2013; 2(1): 65-74.

[9] Mauracher P, Karden E. Dynamic modelling of lead-acid batteries using impédance spectroscopy for parameter idenfificatton. Journal of Power Source.1997; Volume 67: 69-84.

[10] Sunori S, K., \& Juneja, P. K. ANN Controller Design for Lime Kiln Process. Bulletin of Electrical Engineering and Informatics. 2015; 4(4):304-313.

[11] Hannan E, Deistler M. The statistical theory of linear systems. Wiley series in probability and mathematical statistics. John Wiley \& Sons; 1988.

[12] Hemian Lucas Norbert. Wiegman. Battery state estimation and control for power buffering applications. PhD thesis, The University of Wisconsin-Madison. 1999; 216.

[13] Mclntyre M, Burg T, Dawson D, B Xian. Adaptive state of charge Estimator for Battery. Proceedings of the 2006 American Control Conférence Minneapolis. Minnesota, USA. 2006; 14-16

[14] Ghosh S, Maka S. A NARX modeling-based approach for evaluation of insulin sensitivity. Biomedical Signal Processing and Control. 2009; 4(1): 49-56.

[15] Ghosh S. Maka S. Genetic algorithm based NARX model identification for evaluation of insulin sensitivity. Applied Soft Computing. 2011; 11(1): 221-226.

[16] Nader Jamali Soufi Amlashi, Amin Shahsavari, Alireza Vahidifar, Mehrzad Nasirian. Nonlinear System Identification of Laboratory Heat Exchanger Using Artificial Neural Network Model. International Journal of Electrical and Computer Engineering. 2015; Vol 3, No 1. 